\title{
Effect of cerulenin on fatty acid composition and gene expression pattern of DHA-producing strain Colwellia psychrerythraea strain 34H
}

\author{
Xia Wan ${ }^{1,2^{*}}$, Yun-Feng Peng ${ }^{1}$, Xue-Rong Zhou ${ }^{3,4}$, Yang-Min Gong ${ }^{1}$, Feng-Hong Huang ${ }^{1,2}$ \\ and Gabriel Moncalián ${ }^{5^{*}}$
}

\begin{abstract}
Background: Colwellia psychrerythraea $34 \mathrm{H}$ is a psychrophilic bacterium able to produce docosahexaenoic acid (DHA). Polyketide synthase pathway is assumed to be responsible for DHA production in marine bacteria.

Results: Five pfa genes from strain 34H were confirmed to be responsible for DHA formation by heterogeneous expression in Escherichia coli. The complexity of fatty acid profile of this strain was revealed by GC and GC-MS. Treatment of cells with cerulenin resulted in significantly reduced level of C16 monounsaturated fatty acid (C16:1 ${ }^{\Delta 9 t}$, $\left.\mathrm{C} 16: 1^{\Delta 7}\right)$. In contrast, the amount of saturated fatty acids (C10:0, C12:0, C14:0), hydroxyl fatty acids (3-OH C10:0 and

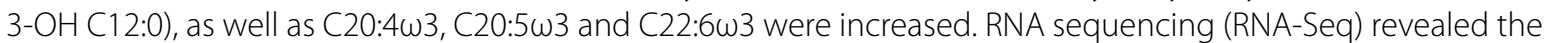
altered gene expression pattern when C. psychrerythraea cells were treated with cerulenin. Genes involved in polyketide synthase pathway and fatty acid biosynthesis pathway were not obviously affected by cerulenin treatment. In contrast, several genes involved in fatty acid degradation or $\beta$-oxidation pathway were dramatically reduced at the transcriptional level.
\end{abstract}

Conclusions: Genes responsible for DHA formation in C. psychrerythraea was first cloned and characterized. We revealed the complexity of fatty acid profile in this DHA-producing strain. Cerulenin could substantially change the fatty acid composition by affecting the fatty acid degradation at transcriptional level. Acyl-CoA dehydrogenase gene family involved in the first step of $\beta$-oxidation pathway may be important to the selectivity of degraded fatty acids. In addition, inhibition of FabB protein by cerulenin may lead to the accumulation of malonyl-CoA, which is the substrate for DHA formation.

Keywords: Cerulenin, DHA, Colwellia psychrerythraea, Fatty acid profile, RNA-seq

\section{Background}

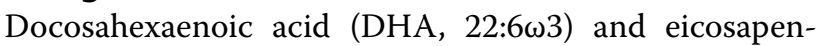
taenoic acid (EPA, 20:5) are the two main commercial omega-3 very long chain polyunsaturated fatty acids

\footnotetext{
*Correspondence: wanxia@oilcrops.cn; gabriel.moncalian@unican.es 1 Key Laboratory of Biology and Genetic Improvement of Oil Crops, Ministry of Agriculture, Oil Crops Research Institute of Chinese Academy of Agricultural Sciences, Wuhan 430062, China

${ }^{5}$ Departamento de Biología Molecular e Instituto de Biomedicina y Biotecnología de Cantabria (IBBTEC), Universidad de Cantabria-CSIC, C/Albert Einstein 22, 39011 Santander, Spain

Full list of author information is available at the end of the article
}

(VLCPUFAs) because of their health benefits. Their beneficial effects in human cardiovascular health, mental health, immune function and infant cognitive development are well documented [1]. In addition, they are precursors for certain hormones and lipid-derived signaling molecules [2]. Such VLCPUFAs can be synthesized in human with a low overall conversion rate using $\alpha$-linolenic acid (ALA, 18:3) as substrate [3], rather than de novo synthesis. Alternatively, food or food additives rich in VLCPUFAs are a good choice to obtain VLCPUFAs directly. 
Commercial VLCPUFAs are mainly produced from fish oils, algae oils or krill oils. Recently, it became more and more attractive to achieve commercial production of VLCPUFAs via metabolic engineering of microbes due to the limited natural resources. Microbes grow fast, are easy to be genetically manipulated and use versatile carbon sources. One of these successful examples, oleaginous yeast Yarrowia lipolytica has been genetically engineered to make EPA at $25 \%$ dry cell weight and at $50 \%$ of total lipids, which is much higher than those of other reported wild-type microbes [4]. In general, VLCPUFAs could be synthesized through conventional fatty acid desaturase/ elongase pathway in algae and animals. There are more than 20 fatty acid desaturases and fatty acid elongases involved in this process [5]. Several key desaturases and elongases, such as $\Delta 6$ desaturase, $\Delta 5$ desaturase, $\Delta 4$ desaturase, $\Delta 9$ elongase, $\Delta 8$ desaturase, $\Delta 5$ elongase, have been transformed into microbes or plants to make them successfully produce VLCPUFAs [6-8]. Alternatively, VLCPUFAs can be synthesized via unconventional polyketide synthase (PKS) pathway as well. This pathway can only be found in narrow species of marine bacteria including Shewanella, Moritella, Vibrio, Photobacterium, Aureispira and Colwellia and some lower eukaryotes [9-12]. PKS system contains a set of essential domains for VLCPUFAs formation including malonyl-CoA:ACP acyltransferase (MAT), acyl carrier protein (ACP), 3-ketoacyl synthase (KS), 3-ketoacyl-ACP reductase (KR), acyltransferase (AT), chain length factor (CLF), enoyl reductase (ER), and 3-hydroxyacyl-ACP dehydrase/isomerase (HD/IS) [13]. Compared to conventional desaturase/elongase pathway, there are only 3-5 genes (pfa gene cluster) responsible for de novo VLCPUFAs synthesis via PKS pathway [12]. This cluster usually contains 1 MAT domain $(p f a A), 1 \mathrm{KR}$ domain ( $p f a A), 1$ CLF domain ( $p f a C), 1$ ER domain $(p f a D)$, 2 KS domains ( $p f a A$ and $p f a C$ ), 3-6 ACP domains ( $p f a A$ ), $1 \mathrm{AT}(p f a B)$ and $2 \mathrm{DH} / \mathrm{IS}$ domains $(p f a C)$ [12]. In addition, PKS pathway was demonstrated to be anaerobic pathway and less NADPH was needed for the whole process compared with that of conventional pathway [14]. Notably, PKS proteins typically show high sequence similarity to enzymes of fatty acid synthase (FAS) but not to any desaturase or elongase [5]. Similarly to bacterial FAS, PKS make use of $p f a$ gene cluster to accomplish the iterative cycle of condensation, reduction, dehydration and further reduction to produce VLCPUFAs. However, the details of synthetic route for VLCPUFAs via PKS and the mechanism of crosstalk between FAS and PKS are still unknown.

Colwellia psychrerythraea strain $34 \mathrm{H}$, is a psychrotrophic gram-negative bacterium isolated from the sediment of Northeast Water Polynya, Greenland. The presence of a $p f a$ gene cluster in the C. psychrerythraea genome suggested that this strain is able to produce DHA
[15]. Recently, C. psychrerythraea $34 \mathrm{H}$ was demonstrated to produce $2.3 \%(\mathrm{w} / \mathrm{w})$ of DHA and trace amount of EPA to the total fatty acids [16]. Cerulenin [(2R,3S)-2,3-epoxy4-oxo-7,10-trans,trans-dodecadienamide] is an FAS inhibitor originally developed as an antifungal antibiotic. It was reported to be an irreversible inhibitor of $\beta$-ketoacyl-ACP synthase I and II activities $[17,18]$ and it could increase the DHA content in Photobacterium, Shewanella and Moritella [19, 20]. However, the mechanism of this process is still unknown. In the present study we describe the DHA production by C. psychrerythraea at different temperatures and with the treatment of cerulenin. Five $p f a$ genes were required for DHA production. Cerulenin treatment resulted in significant increased DHA production, along with the increased levels of medium chain, short chain saturated fatty acids (SFAs) and hydroxyl fatty acids (HFAs), at the expense of medium chain monounsaturated fatty acids (MUFAs). RNA deep sequencing revealed a certain number of genes either up regulated or down regulated, suggesting possible involvement of these genes in regulating of the fatty acid composition.

\section{Methods}

\section{Chemicals, enzymes and kits}

Fatty acid standards, 2-amino-2-methyl-1-propanol, $1 \%$ trimethylchlorosilane in N,O-bis(trimethylsilyl)trifluoroacetamide (BSTFA) and cerulenin were purchased from Sigma-Aldrich (St. Louis, MO). Zwittergent, lysozyme and proteinase $\mathrm{K}$ were purchased from Merck, SigmaAldrich (St. Louis, MO) and Roche Diagnostic GmbH (Mannheim, Germany), respectively. RNA protect reagent, RNeasy Mini Kit and Qiaquick PCR spin columns were purchased from Qiagen (Qiagen, Venlo, The Netherlands). TURBO DNA-free Kit was purchased from Applied Biosystems (Foster City, CA). Ribo-Zero rRNA removal kit for Gram-negative bacteria was purchased from Epicentre. Superscript II Reverse Transcriptase, RNaseH and DNA polymerase I were purchased from Invitrogen (Waltham, MA). PrimeScript RT reagent kit with gDNA eraser and SYBR PremixEx Taq II kit were purchased from TaKaRa (Dalian, Japan).

\section{Strains and growth conditions}

The marine psychrophile bacterium C. psychrerythraea $34 \mathrm{H}$ was purchased from ATCC. This strain was cultured in marine broth 2216 (Difco Laboratories) at the temperature ranging from 0 to $15{ }^{\circ} \mathrm{C}$ without shaking. For solid media, agar was added at $15 \mathrm{~g} / \mathrm{L}$. Cerulenin dissolved in $50 \%(\mathrm{v} / \mathrm{v})$ ethanol was added to culture medium at various concentrations prior to cultivation. To investigate the effect of cerulenin on C. psychrerythraea, cells were cultivated at $10{ }^{\circ} \mathrm{C}$ with shaking at $200 \mathrm{rpm}$ for $24 \mathrm{~h}$ after cerulenin added. 


\section{Fatty acid profile analysis}

Colwellia psychrerythraea $34 \mathrm{H}$ cells were harvested and freeze-dried overnight. Fatty acid methyl esters (FAME) were prepared by direct trans-methylation method [8]. The FAMEs were analyzed by GC-FID (7890A GC, Agilent Technologies, Palo Alto, CA, USA) equipped with a $30 \mathrm{~m}$ BPX70 column (0.25 mm inner diameter, $0.25 \mu \mathrm{m}$ film thickness, SGE, Austin, Texas, USA). Peaks were integrated with Agilent Technologies ChemStation software. GC-MS analysis was essentially performed according to Zhou et al. [21]. Dimethyloxazoline (DMOX) derivatives of FAMEs were prepared according to Fay and Richli [22] to determine the double bond positions. For hydroxyl fatty acids, the FAMEs were further derivatized by trimethylchlorosilane as described [21] to make trimethylsilyl (TMS) derivatives.

\section{Heterogeneous expression of pfa gene cluster in Escherichia coli}

The primers used in this section are listed in Additional file 1: Table S1. Long PCR was carried out with the high fidelity PrimeSTAR GXL DNA Polymerase (Takara Bio) according to manufacturer's instruction. Specifically, the $C$. psychrerythraea pfaABCD sequence was amplified with primers mpfaA-F containing EcoRI restrction site and mpfaD-R containing SalI restrction site, respectively. The resulting DNA fragment of approximately 19 kbp was double digested with EcoRI and SalI, and then subcloned into the pColdI (Takara Bio) to generate pColdI-pfaA-D. $p f a E$ was amplified with primers mpfaEF containing KpnI restriction site and mpfaE-R containing $\mathrm{BamHI}$ restriction site, respectively. The resulting sequence of about 900 bp was double digested with KpnI and $B a m H I$. Then it was inserted into pSTV28 (Takara Bio) to form pSTV28-pfaE. Two compatible vectors were co-transformed into $E$. coli $\mathrm{DH} 5 \alpha$ by electroporation. Positive colonies were confirmed by PCR and sequencing. Transformants were then cultivated in Luria-Bertani (LB) medium with $50 \mathrm{mg} / \mathrm{L}$ ampicillin and $17 \mathrm{mg} / \mathrm{L}$ chloramphenicol. Half $\mathrm{mL}$ of cells pre-cultured at $30{ }^{\circ} \mathrm{C}$ was inoculated into $50 \mathrm{~mL}$ of fresh LB medium supplemented with $50 \mathrm{mg} / \mathrm{L}$ ampicillin and $17 \mathrm{mg} / \mathrm{L}$ chloramphenicol. When $\mathrm{OD}_{600}$ reached at about $0.6,1 \mathrm{mM}$ isopropyl $\beta_{-}^{-} \mathrm{D}^{-}$ 1-thiogalactopyranoside (IPTG) was added into the culture. Then cells were cultivated with shaking at $180 \mathrm{rpm}$ at $15^{\circ} \mathrm{C}$ for $24 \mathrm{~h}$ to allow gene expression. Cells were collected and dried.

RNA extraction, RNA enrichment and preparation of CDNA fragment libraries

Colwellia psychrerythraea 34H was grown in 2216 medium at $10{ }^{\circ} \mathrm{C}$. Overnight grown cells were inoculated into culture flasks containing $3 \mathrm{~mL}$ of 2216 media each, under two treatments with three repeats, One was cultured at $10{ }^{\circ} \mathrm{C}$ (M10), while the other treatment also cultured at $10{ }^{\circ} \mathrm{C}$ but added cerulenin at a final concentration of $12 \mu \mathrm{g} / \mathrm{mL}$ (M10_C). Colwellia psychrerythraea cells were collected at exponentially growing phase (typical optical density at $600 \mathrm{~nm} \sim 0.9$ ), then fixed with two volumes of RNA protect reagent and harvested by centrifugation. The cellular pellet was then lysed with $280 \mu \mathrm{L}$ of lysis buffer $(10 \%$ zwittergent, $15 \mathrm{mg} / \mathrm{ml}$ lysozime and $20 \mathrm{mg} / \mathrm{ml}$ proteinase $\mathrm{K}$ in $\mathrm{TE}$ buffer). Total RNA was isolated using the RNeasy Mini Kit according to manufacturer's instructions. During this RNA purification, the residual DNA in the samples was removed using TURBO DNA-free Kit. NanoDrop ND-1000 spectrophotometer (NanoDrop Technology, Rockland, DE) and Experion Automated Electrophoresis were used to check the quantity and quality of RNA with the RNA StdSens Analysis Kit (Bio Rad). Genomic DNA contamination was tested by quantitative realtime PCR (qPCR) amplification of 16S rRNA using the iO5 iCycler (Bio-Rad). Total RNA samples were stored at $-80^{\circ} \mathrm{C}$ until further use.

Ribo-Zero rRNA Removal Kit was used to remove $16 \mathrm{~S}$ and $23 \mathrm{~S}$ rRNA from total RNA according to the manufacturer's protocol with the exception that no more than $5 \mu \mathrm{g}$ total RNA was treated per enrichment reaction. Each RNA sample was divided into multiple aliquots of $\leq 5 \mu \mathrm{g}$ RNA and separate enrichment reactions were performed for each sample. Enriched mRNA samples were pooled and analyzed on the 2100 Bioanalzyer (Agilent) to asses the reduction of $16 \mathrm{~S}$ and 23S rRNA before the preparation of cDNA fragment libraries. $100 \mathrm{ng}$ of mRNA were treated with the Ambion RNA fragmentation reagents to generate 60-200 nucleotide RNA fragments. After fragmented RNA precipitation, the synthesis of the cDNA first strand was performed using random N6 primers and Superscript II Reverse Transcriptase. Second strand cDNA synthesis followed, using $\mathrm{RNaseH}$ and DNA Polymerase I. Double stranded cDNA was purified using Qiaquick PCR spin columns according to the manufacturer's protocol.

\section{Illumina Genome Analyzer RNA-Seq}

This double-stranded cDNA was then processed for RNA-Seq using the Illumina Genomic DNA Sample Prep kit (Illumina, Inc., San Diego, CA). After loading the amplified material onto independent flow cells, sequencing was carried out by running 36 cycles on the Illumina Genome Analyzer IIx at the BGI (Beijing, China). Illumina RNA-Seq quality scores converted to phred format (http://www.phrap.com/phred/) analyzed the quality of the RNA-Seq reads. 
Mapping to reference genome and annotated genes Colwellia psychrerythraea $34 \mathrm{H}$ genome and gene information were available from NCBI (http://www.ncbi.nlm. nih.gov/genome/1067). After removing low quality reads and the reads containing adaptors, the remaining reads were aligned to the $C$. psychrerythraea $34 \mathrm{H}$ genome using software bowtie2 (version 2.0.2). Reads Per Kilobase per Million mapped reads (RPKM) were calculated for each transcript dividing number of mapped reads by the length of the transcripts and the number of total sequenced reads in this library.

\section{Quantification of pfa transcription in C. psychrerythraea by Quantitative real-time reverse transcription PCR (qRT-PCR)}

After RNA isolation as described in the previous section, first-strand cDNA was synthesized using a PrimeScript RT reagent kit with gDNA eraser. Two microliters of RT product was used as a template for qRT-PCR with SYBR PremixEx Taq II kit. The primers used in this study are listed in Additional file 2: Table S2.The qRT-PCR mixture $(25 \mu \mathrm{L})$ contained $2 \mu \mathrm{L}$ of cDNA, $0.4 \mu \mathrm{M}$ each genespecific primer. The qRT-PCR was performed as follows: $4 \mathrm{~min}$ at $94{ }^{\circ} \mathrm{C}$ followed by 40 cycles of $30 \mathrm{~s}$ at $94{ }^{\circ} \mathrm{C}, 30 \mathrm{~s}$ at $56{ }^{\circ} \mathrm{C}$, and $25 \mathrm{~s}$ at $72{ }^{\circ} \mathrm{C}$, followed by a melting cycle from $56{ }^{\circ} \mathrm{C}$ to $94{ }^{\circ} \mathrm{C}$ to check for amplification specificity. To assess for DNA contamination, RNA samples were run without reverse transcriptase. The constitutively expressed 16S ribosomal RNA gene was used as an internal control for relative quantification, using the comparative threshold method.

\section{Results}

\section{Optimal growth condition of C. psychrerythraea34H for DHA production}

Colwellia psychrerythraea $34 \mathrm{H}$ is a deep-sea bacteria containing a VLCPUFA Pfa synthase cluster according to its genomic sequence [15]. The 5 pfaEABCD genes responsible for de novo bacterial PUFA biosynthesis encode large, multi-domain protein complexes similar to type I iterative fatty acid and polyketide synthases [10]. PfaB has been described to be the chain length determinant in the VLCPUFA synthesis [23]. Colwellia psychrerythraea $\mathrm{PfaB}$ sequence showed $52 \%$ identity with $\mathrm{PfaB}$ of DHA-producing M. marina and $27 \%$ identity with PfaB of EPA-producing S. baltica, and was expected to allow the production of DHA [12]. In fact, the DHA production has been recently demonstrated [16]. In order to optimize the DHA production, we have tested different growth temperatures for C. psychrerythraea $34 \mathrm{H}$. As shown in Fig. 1a, C. psychrerythraea $34 \mathrm{H}$ optimal growth temperature was found to be at $10{ }^{\circ} \mathrm{C}$. Higher and lower growth temperatures resulted

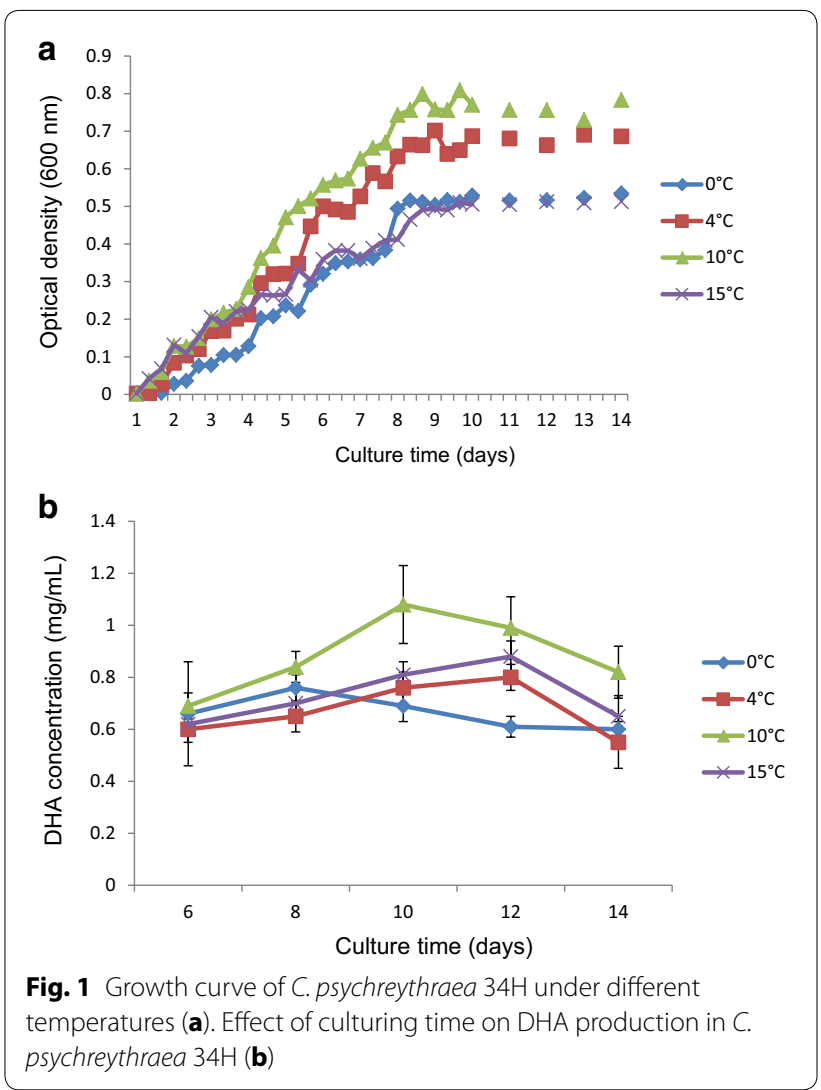

in longer generation times (Fig. 1a). DHA production by C. psychrerythraea $34 \mathrm{H}$ grown at various temperatures was also analyzed by GC. Maximum DHA production was observed at $10{ }^{\circ} \mathrm{C}$ for 10 days, with accumulation of $2.3 \%$ DHA of the total fatty acids (Fig. 1b; Table 1). This DHA production was slightly lower than the production reported for M. marina (5.9\%). M. marina PfaA contains six ACP domains, while C. psychrerythraea only has five ACP domains. It was reported that the number of ACP domains was directly related to the capacity of VLPUFA synthesis [24].

\section{Five genes involved in PKS pathway are essential to DHA formation}

Genes involved in PKS pathway including $p f a A$, $p f a B$, $p f a C, p f a D$ (2'-nitropropane dioxygenase) and $p f a E$ (4'-phosphopantetheinyl transferase), denoted as CPS_ RS13885, CPS_RS13880, CPS_RS13875, CPS_RS13865 and CPS_RS13895 in the genome sequence, have high sequence similarity to corresponding $p f a$ genes from other DHA-producing bacteria [10]. The function of these five genes was further confirmed by heterologous co-expression of them in E. coli. In the control E. coli transformant containing either of two empty vectors pColdI or pSTV28, 16:0, 16:1 and 18:1 were present as 


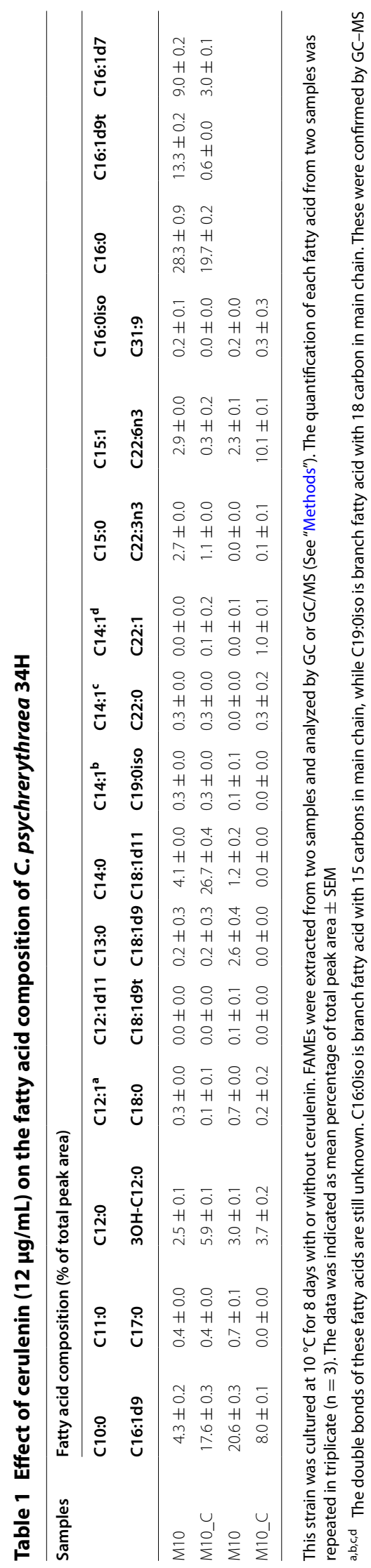


major components according to GC result. In contrast, an additional peak identified as DHA was detected in transformants harboring $p f a A B C D E$ from strain $34 \mathrm{H}$. The DHA content was about $1.2 \%$ of the total fatty acids (Fig. 2).

\section{Occurrence of multiple components of monounsaturated,} polyunsaturated and hydroxyl fatty acids

GC analysis of total fatty acid methyl esters (FAMEs) revealed the complexity of fatty acid profile in C. psychrerythraea $34 \mathrm{H}$ cells grown at $10{ }^{\circ} \mathrm{C}$ (Fig. 3). The major fatty acids were C16 fatty acids (saturate C16:0 and monosaturated $\mathrm{C} 16: 1$ ), followed by $\mathrm{C} 14$ fatty acids and VLCPUFA DHA C22:6. Multiple monounsaturated $\mathrm{C} 16$ and $\mathrm{C} 18$ fatty acid peaks were also detected. These peaks were confirmed as $C 16: 1^{\Delta 9 t}, C 16: 1^{\Delta 7 c}, C 16: 1^{\Delta 9 c}$, $\mathrm{C} 16: 1^{\Delta 11 \mathrm{c}}, \mathrm{C} 18: 1^{\Delta 9 \mathrm{t}}, \mathrm{C} 18: 1^{\Delta 7 \mathrm{c}}, \mathrm{C} 18: 1^{\Delta 9 \mathrm{c}}, \mathrm{C} 18: 1^{\Delta 11 \mathrm{c}}$, both by GC analysis comparing to known standards, or by GCMS analysis to confirm the mass spectra and determine the double bond position. Three major $\mathrm{C} 16: 1$ peaks were detected, eluted at the corresponding retention time of FAMEs prepared from commercial available fatty acids $\mathrm{C} 16: 1^{\Delta 9 \mathrm{t}}, \mathrm{C} 16: 1^{\Delta 6 \mathrm{c}}, \mathrm{C} 16: 1^{\Delta 9 \mathrm{c}}$, with an additional shoulder peak that appeared in trace amount after $C 16: 1^{\Delta 9 c}$

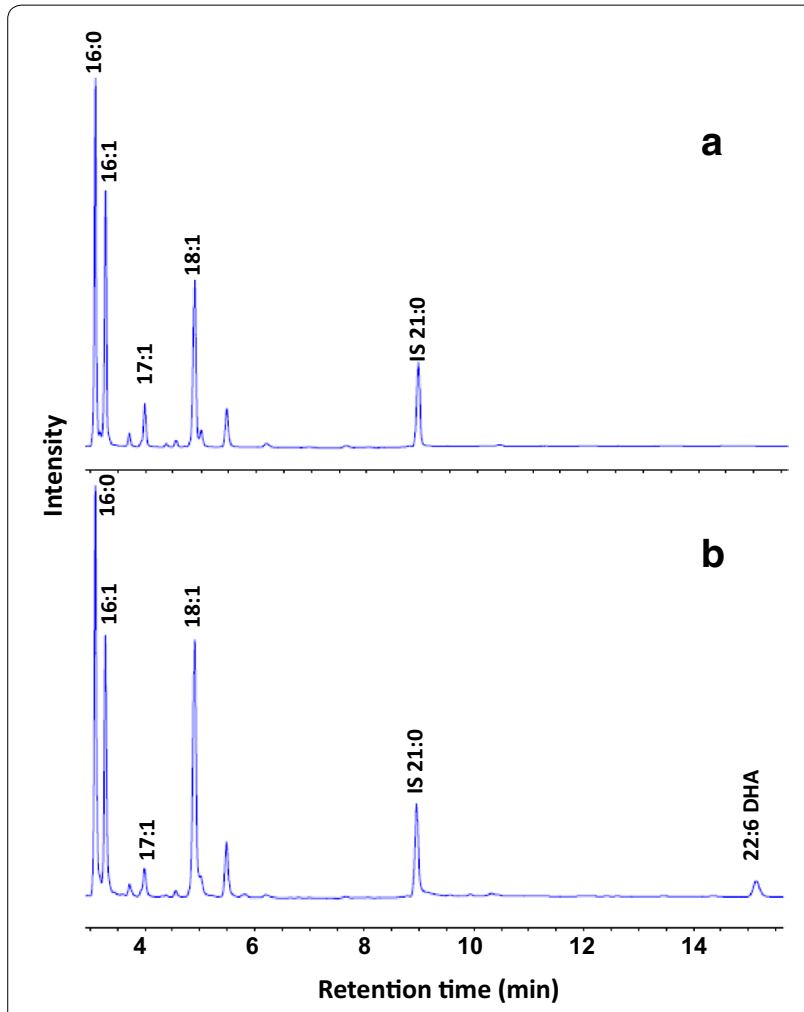

Fig. 2 Confirmation of the functions of five pfa genes in E. coli. GC analysis of FAMEs from the E. coli harboring $p f a A B C D E(\mathbf{b})$ or empty vector $(\mathbf{a})$
FAME (Additional file 3: Figure S1). GC-MS analysis of these peaks confirmed all four peaks showed typical C16:1 FAME mass spectra, although with different retention times. While the presence of $C 16: 1^{\Delta 9 t}$ and $C 16: 1^{\Delta 9 c}$ was confirmed by GC-MS, the second C16:1 peak was identified as $\mathrm{C} 16: 1^{\Delta 7}$ rather than $\mathrm{C} 16: 1^{\Delta 6}$ (Additional file 4: Figure S2). The tiny shoulder peak was identified as $C 16: 1^{\Delta 11 c}$. Similarly, three small $C 18: 1$ peaks were detected, and confirmed as $\mathrm{C} 18: 1^{\Delta 9 t}, \mathrm{C} 18: 1^{\Delta 9 \mathrm{c}}, \mathrm{C} 18: 1^{\Delta 11 \mathrm{c}}$ by GC analysis comparing to the FAMEs prepared from commercial available $\mathrm{C} 18: 1^{\Delta 9 \mathrm{t}}, \mathrm{C} 18: 1^{\Delta 9 \mathrm{c}}, \mathrm{C} 18: 1^{\Delta 11 \mathrm{c}}$ fatty acid standards (Additional file 5: Figure S3) or by GCMS (data not shown). Two hydroxyl fatty acids were also detected, being identified as 3-OH C10:0 and 3-OH C12:0 (Additional file 6: Figure S4). The position of the hydroxyl group was confirmed with TMS derivatives (Additional file 7: Figure S5) as described in "Methods" section. Trace amounts of branch fatty acids were also identified (Additional file 8: figure S6).

In addition to DHA, trace amounts of other VLCPUFA including $\mathrm{C} 20: 4 \omega 3$ and $\mathrm{C} 20: 5 \omega 3$ were also found (Additional file 9: Figure S7). The identities of these components were confirmed by GC analysis comparing the FAME standards, or by GC-MS analysis of FAMEs and/ or their DMOX derivatives.

\section{Effect of cerulenin treatment on fatty acid composition of C. psychrerythraea $34 \mathrm{H}$}

Colwellia psychrerythraea cells grown at $10{ }^{\circ} \mathrm{C}$ treated with the FAS inhibitor cerulenin resulted in a significant fatty acid profile change. The $\mathrm{C} 16$ and $\mathrm{C} 18$ monounsaturated fatty acids (MUFAs) especially $\mathrm{C} 16: 1^{\Delta 9 t}, \mathrm{C} 16: 1^{\Delta 7}$, were greatly reduced after the treatment with cerulenin (Table 1). C16:1 $1^{\Delta 9 t}$ was decreased from 13.3 to $0.6 \%$, while $\mathrm{C} 16: 1^{\Delta 7 \mathrm{c}}$ decreased from 9.0 to $3.0 \%$. C16:1 ${ }^{\Delta 9 \mathrm{c}}$ was decreased from 20.6 to $8.0 \%$. C18 MUFAs reduced from total of $2.7 \%$ to undetectable level. In contrast, increased levels of short chain or medium chain saturated fatty acids (SFAs) C10:0, C12:0 and C14:0, hydroxyl fatty acids 3-OH C10:0 (trace amount) and 3-OH C12:0, as well as

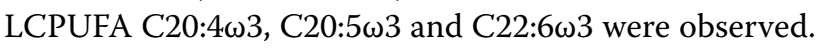
At the optimal concentration of $12 \mu \mathrm{g} / \mathrm{mL}$, cerulenin

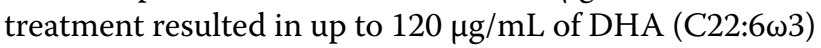
and $10.1 \%$ to the total fatty acids (Fig. 4; Table 1 ).

Transcriptome analysis of C. psychrerythraea after cerulenin treatment revealed the differential response of pathways

RNA-seq was used to analyze the expression patterns of C. psychrerythraea treated with or without $12 \mu \mathrm{g} / \mathrm{mL}$ of cerulenin. To compare the transcriptional activation or repression by cerulenin, normalized RPKM were calculated as described in materials and methods for each of 


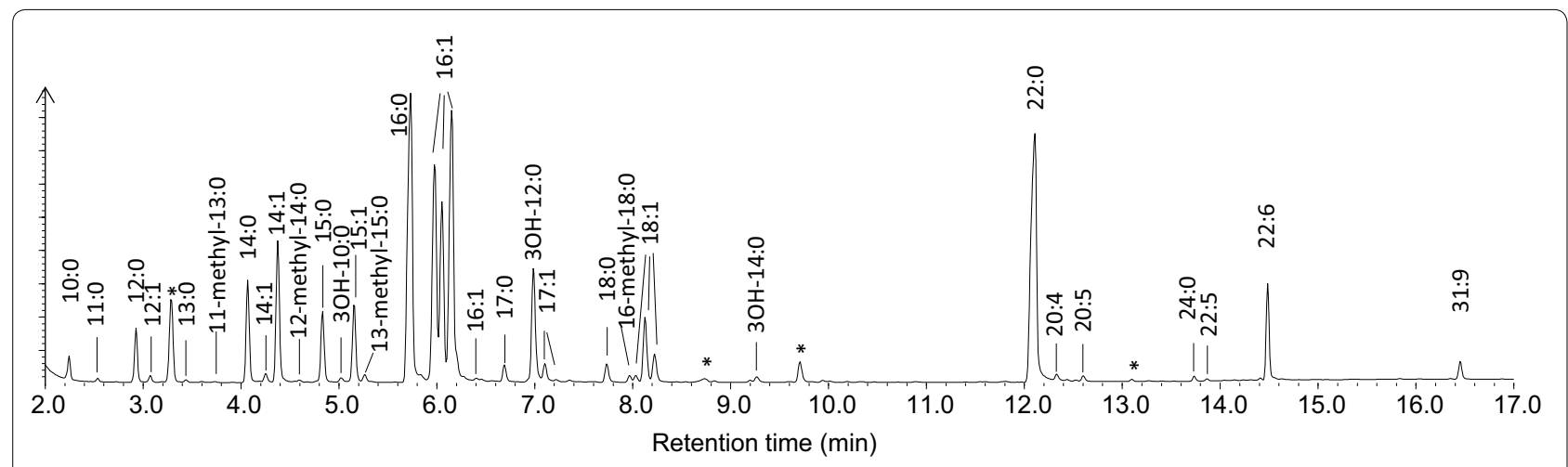

Fig. 3 Gas chromatogram of C. psychrerythraea 34H total FAMEs. *indicated the unknown peaks

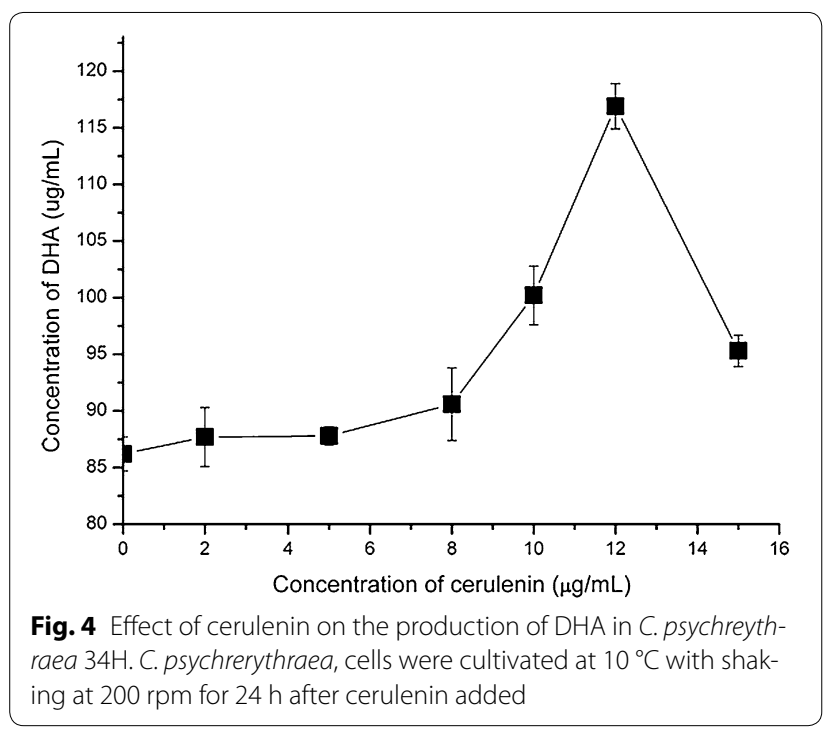

the 5048 annotated C. psychrerythraea genes. As many as 240 genes were up-regulated by cerulenin treatment (twofold or greater, M10_C vs M10), while other 193 genes were down-regulated (twofold or greater, M10_C vs M10) (Additional file 10: Figure S8). For an overview of the metabolic changes that occurred after the cerulenin treatment we identified the KEGG pathways [25] corresponding to the up- or down-regulated genes. As shown in Fig. 5, catabolic pathways such as carbon metabolism, amino acid degradation pathways, TCA cycle or glucolysis pathways were up-regulated by cerulenin. On the other hand, amino acid biosynthesis, ribosomal genes or $\mathrm{ABC}$ transporters were down-regulated.

\section{Expression of most genes involved in PKS and fatty acid biosynthesis pathway was not affected by cerulenin} Despite the increased level of DHA under these conditions, supplementation of cerulenin did not increase the

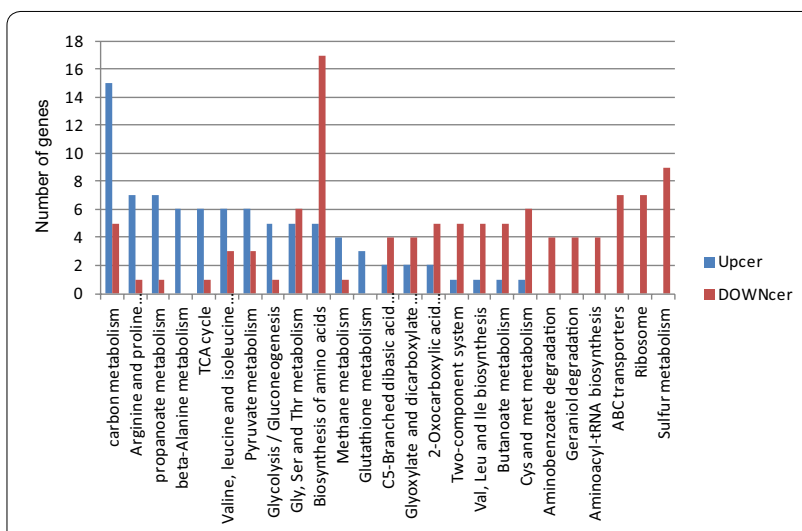

Fig. 5 Summary of KEGG annotations for the number of genes. Distribution of up-regulated genes (Upcer) and down-regulated genes (DOWNcer) in the cerulenin treated sample

expression of the five $p f a$ genes involved in PUFAs biosynthesis (Table 2). This result was further confirmed by qRT-PCR (Additional file 11: Table S3). Moreover, genes involved in FA biosynthesis were not obviously affected by supplementation of cerulenin (Table 2). RNA-seq analysis showed that the expression of $f a b B$ (CPS_RS17030), reported to be responsible for the elongation of unsaturated fatty acid and proven to be the target for cerulenin [18], was not inhibited at the mRNA level. Another gene CPS_RS00390 encoding for cis/tran isomerase that could alter the ratio of cis- to trans-esterified fatty acids in phospholipids [15] was not changed at RNA level, although the trans monounsaturated fatty acids $\left(\mathrm{C} 16: 1^{\Delta 9 \mathrm{t}}\right.$ and $\left.\mathrm{C} 18: 1^{\Delta 9 \mathrm{t}}\right)$ were greatly reduced.

However, several genes involved in fatty acid degradation, such as fadD1 (CPS_RS05300) and fadB (CPS_ RS01765), were down-regulated. Besides, the genes encoding for acyltransferases involved in polar lipid and neutral lipid formation, including glycerol-3-phosphate acyltransferase (CPS_RS10220, CPS_RS19415, CPS_RS00605), 
Table 2 Differential expression of genes involved in fatty acid metabolism in C. psychrerythraea without or with $12 \mu \mathrm{g} /$ $\mathrm{mL}$ cerulenin at $10^{\circ} \mathrm{C}$

\begin{tabular}{|c|c|c|c|}
\hline Locus ID & Gene ID & Gene description & Fold change $\left(\log _{2}\right)$ \\
\hline \multicolumn{4}{|c|}{ Gene involved in fatty acid biosynthesis } \\
\hline CPS_RS06985 & accA & acetyl-CoA carboxylase subunit A & 0.28 \\
\hline CPS_RS04205 & $\mathrm{acc} B$ & acetyl-CoA carboxylase subunit B & 0.47 \\
\hline CPS_RS04200 & accC & acetyl-CoA carboxylase subunit $C$ & 0.39 \\
\hline CPS_RS17005 & $\mathrm{acc} D$ & acetyl-CoA carboxylase subunit D & -0.31 \\
\hline CPS_RS14645 & fabA & 3-hydroxydecanoyl-ACP dehydratase, isomerase & -0.21 \\
\hline CPS_RS17030 & fabB & 3-ketoacyl-ACP synthase I & 0.96 \\
\hline CPS_RS10240 & fabF & 3-ketoacyl-ACP synthase II & 0.41 \\
\hline CPS_RS09575 & $\mathrm{fabH}$ & 3-ketoacyl-ACP synthase III & 0.38 \\
\hline CPS_RS10225 & fabD & malonyl-CoA:ACPtransacylase & 0.95 \\
\hline CPS_RS07145 & fabG1 & 3-ketoacyl-ACP reductase & 0.01 \\
\hline CPS_RS10230 & fabG2 & NADPH-dependent 3-ketoacyl-ACPreductase & -0.03 \\
\hline CPS_RS06960 & fabZ & 3-hydroxyacyl-ACP dehydratase & -0.06 \\
\hline CPS_RS01535 & $\mathrm{fabR}$ & DNA-binding transcriptional repressor & -0.04 \\
\hline \multicolumn{4}{|c|}{ Gene involved in fatty acid degradation } \\
\hline CPS_RS01760 & fadA & 3-ketoacyl-CoA thiolase & -0.57 \\
\hline CPS_RS01765 & $\mathrm{fadB}$ & 3-hydroxyacyl-CoA dehydrogenase & -0.92 \\
\hline CPS_RS05300 & fadD1 & Long chain fatty acid-CoA ligase & -1.51 \\
\hline CPS_RS15290 & fadD2 & Long chain fatty acid-CoA ligase & 0.28 \\
\hline CPS_RS10085 & fadE & Acyl-CoA dehydrogenase & 0.40 \\
\hline CPS_RS04110 & $\mathrm{fadH}$ & 2,4-dienoyl-CoA reductase & 0.55 \\
\hline CPS_RS15575 & fadR & Fatty acid metabolism regulator & -0.39 \\
\hline \multicolumn{4}{|c|}{ Gene involved in fatty acid modification or lipid metabolism } \\
\hline CPS_RS00390 & cti & fatty acid cis/trans isomerase & 0.11 \\
\hline CPS_RS10220 & plsX & glycerol-3-phosphate acyltransferase & -0.30 \\
\hline CPS_RS19415 & plsY & glycerol-3-phosphate acyltransferase & 0.31 \\
\hline CPS_RS00605 & GPAT & glycerol-3-phosphate acyltransferase & 0.21 \\
\hline CPS_RS04525 & LPAAT & 1-acyl-sn-glycerol-3-phosphate acyltransferase & 0.06 \\
\hline CPS_RS21310 & LPAAT & 1-acyl-sn-glycerol-3-phosphate acyltransferase & -0.08 \\
\hline CPS_RS04435 & & acyltransferase & 0.02 \\
\hline CPS_RS08065 & & acyltransferase & 0.25 \\
\hline CPS_RS14560 & & acyltransferase & 0.55 \\
\hline CPS_RS21745 & & acyltransferase & 0.52 \\
\hline CPS_RS22505 & & acyltransferase & 0.70 \\
\hline CPS_RS22520 & & acyltransferase & -0.20 \\
\hline \multicolumn{4}{|c|}{ Genes involved in PKS pathway } \\
\hline CPS_RS13885 & $\mathrm{pfaA}$ & polyunsaturated fatty acid synthase & -0.58 \\
\hline CPS_RS13880 & $\mathrm{pfaB}$ & polyunsaturated fatty acid synthase & -0.37 \\
\hline CPS_RS13875 & $\mathrm{pfaC}$ & polyunsaturated fatty acid synthase & 0.03 \\
\hline CPS_RS13865 & $\mathrm{pfaD}$ & polyunsaturated fatty acid synthase & 0.60 \\
\hline CPS_RS13895 & $\mathrm{pfaE}$ & 4'-phosphopantetheinyl transferase & -0.51 \\
\hline
\end{tabular}

Fold change ( $\log _{2}$ values) in transcript levels under specified conditions as determined by RNA-seq. Transcript abundance obtained from RNA-seq data is indicated as RPKMs (See "Methods" section)

1-acyl-sn-glycerol-3-phosphate acyltransferase (CPS RS04525, CPS_RS21310) were not affected. Three out of six genes for other annotated acyltransferases (CPS_RS04435,
CPS_RS08065, CPS_RS14560,CPS_RS21745,CPS_RS22505 and CPS_RS22520) had slight increased expression level. Their functions are to be further investigated. 


\section{Top up-regulated genes by cerulenin}

The most up-regulated genes in the cerulenin-treated cells were found to be the operons containing oxidoreductase genes. CPS_RS06205 gene, coding for an oxidoreductase, FAD/FMN-binding protein was dramatically upregulated $\left(\log _{2}\right.$ fold change was 7.31$)$ (Additional file 12: Table S4). We carried out the structural prediction and modeling analysis of CPS_RS06205 using the Protein Homology/analogY Recognition Engine (PHYRE) Web server [26]. As shown in Fig. 6a, CPS_RS06205 was found to be homolog to the Old Yellow Enzymes (OYEs, NCBI accession number AAN56390), flavin-dependent enoate reductases (EC 1.6.99.1) that catalyze the stereoselective hydrogenation of activated double bonds of different alkenes [27]. Another highly up-regulated gene by cerulenin was CPS_RS04135. CPS_RS04135 belongs to the crotonyl-CoA carboxylase-reductase (CCR) family, involved in extender-unit biosynthesis of polyketides [28]. Moreover, CPS_RS07445, upregulated 11-fold, codes for a short chain dehydrogenase/reductase family oxidoreductase (Fig. 6b). CPS_RS07440, upregulated sixfold, codes for a FMN-dependent NADH-azoreductase. Genes CPS_RS18085 (11-fold) and CPS_RS18095 (fourfold), also code for allantoate amidohydrolase and NADPH-dependent F420 reductase, respectively (Additional file 12: Table S4).

\section{Discussion}

\section{Complexity of C. psychrerythraea fatty acid profile}

Although the major fatty acids in C. psychrerythraea $34 \mathrm{H}$ cells were C16 fatty acids, the very complicated fatty acid

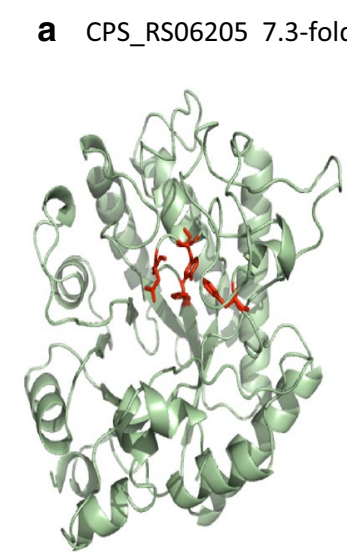

Old Yellow Enzyme Reductase OYE4 b CPS_RS07445 3.5-fold

short-chain dehydrogenase /reductase (SDR)

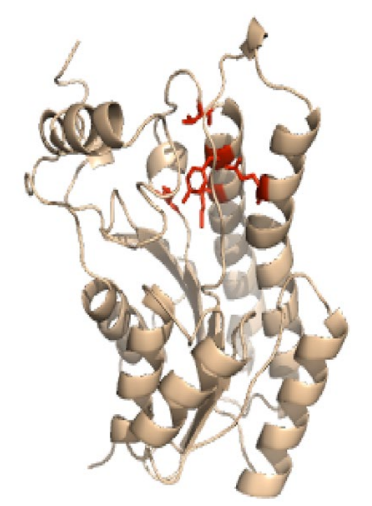

Fig. 6 Structural prediction analysis of the CPS_RS06205 (a) and CPS_RS07445 (b) revealed their similarity to old yellow enzyme reductase OYE4 and short chain dehydrogenase/reductase, respectively profile was uncovered in this work. These FAs included short and medium chain SFAs, odd number carbon length FAs, short and medium chain MUFAs, multiple component of monounsaturated C16:1 or C18:1, branch fatty acids, PUFAs and 3-HFAs, although some of the components had very low levels. The existence of these components was confirmed by GC-MS analysis. The occurrence of trans monounsaturated C16:1 and PUFAs in $34 \mathrm{H}$ has also been recently reported [16]. Under our experimental conditions, we detected more components that were not reported previously. These included trace amounts of C11:0, C13:0, three isomers of C14:1, 3-OH C10:0, C18: $1^{\Delta 9 \text { trans }}$, and branch fatty acids C16:0iso and C19:0iso. Such fatty acid profile could indicate the complexity of fatty acid synthesis and modification pathways in strain $34 \mathrm{H}$.

We showed that the fatty acid profile was differentially affected by cerulenin treatment. Cerulenin, a specific inhibitor of condensing enzymes, had been reported to inhibit MUFA synthesis but not PUFA synthesis in deep-sea bacteria M. marina [19]. This is consistent to our result that C16:1 and C18:1 MUFAs were significantly decreased, especially the trans fatty acids, while the VLCPUFA DHA increased. We also showed the enhanced levels of C10:0, C12:0, C14:0 and 3-hydroxy C12:0 after cerulenin treatment (Table 1 ). The condensing enzymes principally extend the fatty acid chain length by two carbons in each fatty acid synthesis cycle. Inhibition of the condensing enzymes by cerulenin could explain the accumulation of shorter chain length fatty acid, and decreased the longer chain length fatty acids. Indeed, C16:0 and C18:0 FAs were both reduced after cerulenin treatment. However, the significant increase of C14:0 levels might suggest the condensing step from C12:0 to C14:0 was not inhibited, same as the step from C8:0 to C10:0. It is possible that cerulenin might inhibit these steps differently, or these steps might be catalyzed by different condensing enzyme isomers. In addition, C16 and C18 MUFAs were decreased. Escherichia coli possesses at least three condensing enzymes: FabH selectively uses acetyl-CoA to initiate the fatty acid synthesis pathway, and FabB/FabF carry out the subsequent rounds of fatty acid elongation which condense malonyl-ACP with acyl-ACP to extend the acyl chain by two carbons [29]. FabF is confirmed to be the sole condensing enzyme for subsequent elongation step in this strain [30]. In contrast, Bacillus subtilis contains two FabH isozymes (FabHA and FabHB) which differ from E. coli FabH in that they could initiate both the straight and branched chain fatty acid synthesis. Similar to E. coli, only one initial condensing enzyme FabH (CPS_RS09575) and two other condensing enzymes, FabB (CPS_RS17030) and FabF (CPS_RS10240), were denoted in the genome of $34 \mathrm{H}$. 


\section{No effect of PKS pathway at transcriptional level}

In order to better understand the effect of cerulenin on the fatty acid level change, we performed the transcriptome analysis. To our surprise, there was no obvious change of PUFA PKS pathway at the transcription level after cerulenin treatment. The five $p f a$ genes were confirmed to be essential to DHA production in strain $34 \mathrm{H}$ by heterologous co-expression in E. coli. However, all these five genes did not increase the expression level after cerulenin treatment, although the DHA level was significantly increased. This might suggest the increase of DHA content in C. psychrerythraea was not regulated by $p f a$ gene cluster at the transcriptional level when cells were treated with cerulenin. It was reported that cultivation of P. profundum strain SS9 at elevated hydrostatic pressure or reduced temperature did not increase $p f a$ gene expression despite the resulting increase in percentage composition of EPA under these conditions [31]. We proposed that there could be some other genes or transcriptional factors related to the increase of DHA accumulation. Another explanation could be that the availability of malonyl-CoA for PKS pathway is higher after cerulenin treatment.

Besides, $p f a$ genes are regulated by $\mathrm{PfaR}$. PfaR is predicted to be an inactive transcriptase that does not seem to be affected for any effectors. Therefore, it has sense that cerulenin affecting fatty acid profile does not affect PfaR binding and thus does not affect PfaABCD transcription. A BLAST search against current databases using the sequence of $\mathrm{PfaR}$ revealed more than 100 putative PfaR homologs in marine bacteria, but not in $M$. marina (data not shown). In fact the PfaRABCD cluster is conserved in all these marine bacteria. Moreover, we carried out structural prediction and modeling of PfaR using the PHYRE. 263 residues (91\%) of PfaR could be modeled at $>90 \%$ accuracy using structure 4FCY (crystal structure of the bacteriophage mu transpososome) as a model, although the sequence identity between PfaR and $4 \mathrm{FCY}$ is only $14 \%$ (unpublished data). MuA, like many other DNA transposases, and retroviral integrases share a conserved RNaseH-like or "DDE" catalytic domain, named for the $3 \mathrm{Mg}^{2+}$ binding carboxylates in their active sites. However, these catalytic residues are not conserved in PfaR. Thus, we think PfaR is not an active transposase but rather a transcriptional regulator.

Similarly, the gene CPS_RS00390 coding for a cis/ trans isomerase that could alter the ratio of cis- to transesterified fatty acids in phospholipids was not obviously changed at RNA level, although the trans monounsaturated fatty acids $\left(\mathrm{C} 16: 1^{\Delta 9 t}\right.$ and $\left.\mathrm{C} 18: 1^{\Delta 9 t}\right)$ were greatly reduced. Moreover, considering that the reduction level of $C 16: 1^{\Delta 9 t}$ was much higher than that of $C 16: 1^{\Delta 9 c}$, a more complicated effect could exist rather than a simple effect of CPS_RS00390 expression level.

\section{Down-regulation of some genes involved in fatty acids degradation}

Bacterial FASII system contained basically the same set of enzymatic domains as PKS [30]. Although FAS and PKS used the same substrates, acetyl-CoA and manoly-CoA, the final products were quite distinctive. In general, FAS was responsible for the essential short and medium chain fatty acids while PKS was only capable of production of VLCPUFAs [32]. Until now, the mechanism of cross talk between FAS and PKS was still unknown. FAS pathway can be classified into fatty acids biosynthesis pathway and fatty acids degradation pathway. As shown in Table 2, most of the genes involved in the fatty acid biosynthesis pathway were not obviously affected in the presence of cerulenin. Notably, one of those genes, $f a b B$ encoding for 3-ketoacyl-ACP synthase, was responsible for the elongation of unsaturated fatty acids. FabB activity was demonstrated to be irreversibly inhibited by cerulenin [18]. Although the content of MUFAs, such as C16:1 ${ }^{\Delta 9 t}$, $\mathrm{C} 16: 1^{\Delta 7 \mathrm{c}}$ and $\mathrm{C} 16: 1^{\Delta 9 \mathrm{c}}$ was indeed decreased (Table 1), the transcriptional expression of $f a b B$ was not downregulated in the presence of cerulenin in this test, but slightly up-regulated (Table 2). It was also demonstrated that transcription of the operon containing the $f a b F$ gene from Bacillus subtilis increased eightfold in response to cerulenin treatment [33]. We speculated that the minor increase of $f a b B$ or $f a b F$ might be to compensate the low activity of $\mathrm{FabB} / \mathrm{FabF}$ protein.

In contrast, two genes ( $f a d B$ and $f a d D 1$ ) involved in the fatty acid degradation pathway were dramatically downregulated (Table 2). In the first step of long chain fatty acid degradation, fatty acids would be transported across the cell membrane via a transport and acyl-activation mechanism. Gene $f a d D$ encoding an inner membranebound acyl-CoA synthase was involved in this process. In general, there is only one acyl-CoA synthase in $E$. coli with broad substrate specificity [30]. However, in $C$. psychrerythraea, two genes $f a d D 1$ and $f a d D 2$, were both denoted as acyl-CoA synthases. Only fadD1 was dramatically down-regulated while $f a d D 2$ was slightly up-regulated (Table 2). Therefore, two FadD enzymes might have different substrate specificities based on the changed fatty acids profile. This result awaits further investigation. The other down-regulated gene $f a d B$ was thought to encode for enoyl-CoA hydratase/3-hydroxyacyl-CoA dehydrogenase. This enzyme converted enoyl-CoA to 3-ketoayl-CoA via 3-hydroxylacyl-CoA through hydration and oxidation [30]. Both $f a d D$ and $f a d B$ played critical roles in fatty acid degradation. 
The final degradation of acyl-CoA is achieved by $\beta$-oxidation pathway. In $E$. coli, gene fadE encoding for acyl-CoA dehydrogenase, was demonstrated to be responsible for the conversion of acyl-CoA to enoyl-CoA. This gene was also thought to be the only acyl-CoA dehydrogenase gene in E. coli [34]. According to the genome sequence of strain 34H, CPS_RS10085, denoted as fadE, codes for an acyl-CoA dehydrogenase. However, there were six other genes denoted as acyl-CoA dehydrogenase as well in this strain (Additional file 13: Figure S9). Notably, only CPS_RS02980 and CPS_RS10085 were up-regulated while the others including CPS_RS10075, CPS_RS05245, CPS_RS12125, CPS_RS20065 and CPS_ RS02940 were down-regulated (Additional file 13: Figure S9). The expansion of acyl-CoA dehydrogenase gene family might be important to the selective oxidation of short (C4-C6), medium (C8-C14), long (C16-C18) and very long $(>\mathrm{C} 20)$ chain fatty acids. This could be one of the reasons led to the higher accumulation of DHA after cerulenin treatment.

\section{Other top regulated genes by cerulenin might contribute to the changes of fatty acid profile in strain $34 \mathrm{H}$}

Except for fatty acid metabolism related genes, other significantly regulated genes were also investigated. Three of the top 20 up-regulated genes are TetR family regulators (CPS_RS06200, CPS_RS07325 and CPS_RS07450, Additional file 12: Table S4). They belong to one-component systems in which a single polypeptide contains both a sensory domain and a DHA-binding domain [35]. They are widely associated with many biological processes, such as antibiotic resistance and fatty acids synthesis [36, 37]. These proteins have several homologs in the Protein Data Bank (PDB). One of them is FadR. FadR binds one lauroyl (C12)-CoA molecule per FadR monomer, with its acyl chain moiety in the center of the FadR molecule, enclosed within a tunnel-like substrate-binding pocket surrounded by hydrophobic residues, and the CoA moiety interacting with basic residues on the protein surface [36]. Recently, fadR was demonstrated to be an important transcriptional factor that was capable of positively control the $f a b A$ and $f a b B / F$ genes in E. coli [37]. Overexpression of E. coli FadR could dramatically increase the fatty acid titer by 7.5 -fold over wild-type strains. Moreover, the unsaturated fatty acids including 14:1, 16:1 and 18:1 were increased [37]. Besides, FadR was demonstrated to dissociate with $\mathrm{C} 16-\mathrm{C} 18$ fatty acids but not with C10 fatty acids [38]. Thus, cerulenin is not expected to affect FadR regulation.

The other most up-regulated gene was CPS_RS06205. The predicted protein structure of this gene showed high similarity to OYEs. OYEs have been found to be highly expressed in the EPA producing strain S. oneidensis [39].
The expression of OYE in S. oneidensis was observed in the absence of cerulenin. Thus, CPS_RS06205 upregulation seems to be related to the metabolic changes that drive DHA accumulation and not to be the cerulenin effect itself.

CPS_RS04135 denoted as a CCR family gene, was dramatically up-regulated as well. It could reduce double bond in the accumulated MUFAs and other fatty acids to produce the extender units needed by PKS for DHA synthesis. Therefore, the accumulation of precursors might be one possible reason for DHA increase in cerulenintreated cells. On the other side, CPS_RS07330 (14-fold) is homolog to the multidrug efflux protein AcrB. AcrA/B system is controlled by the TetR-like protein ArcR. This efflux system could be upregulated to cope with the toxicity of cerulenin. The ability to reduce the dye 2,3,5-triphenyltetrazolium chloride (TTC) has been shown to be directly associated with EPA production in marine Gram-negative bacteria in solid or liquid medium [40]. The up-regulated oxidoreductases could be responsible of this reduction. Moreover, detoxification of $\mathrm{H}_{2} \mathrm{O}_{2}$ is other characteristic of PUFA producing deep-sea bacteria [41] that could also be due to the expression of different reductases.

Although C. psychrerythraea 34H cell produces DHA, it can only grow at relatively low temperature, and the transformation system for genetic modification has not been set up. However, this strain synthesizes DHA via PKS pathway. Compared to conventional desaturase/ elongase pathway, PKS is an anaerobic pathway and less NADPH was needed for the whole process. More important, unlike desaturase/elongase pathway, the final products of PKS are only VLCPUFAs. Our current work is to seek and investigate good gene candidates from microbes harboring PKS pathway. These genes can be engineered into other expression systems such as oleaginous yeast $Y$. lipolytica [4] to produce high levels of VLCPUFAs.

\section{Conclusions}

In summary, the complexity of psychrophilic C. psychrerythraea fatty acid profile was revealed by GC or GC-MS. Cerulenin treatment of this strain resulted in enhanced accumulation of short and medium chain saturated fatty acids, 3-hydroxy fatty acids, and DHA, at the expense of $\mathrm{C} 16$ and $\mathrm{C} 18$ saturated and MUFAs. Five pfa genes involved in PKS pathway were demonstrated to be responsible for DHA production by heterogeneous expression in E. coli. However, genes involved in polyketide synthase pathway and fatty acid biosynthesis pathway were not obviously affected by cerulenin treatment. In contrast, several genes involved in fatty acid degradation or $\beta$-oxidation pathway were dramatically reduced at the transcriptional level. The results suggested 
that acyl-CoA dehydrogenase gene family involved in the first step of $\beta$-oxidation pathway may be important to the selectivity of degraded fatty acids. In addition, inhibition of FabB protein by cerulenin may lead to the accumulation of malonyl-CoA, which is the substrate for DHA formation.

\section{Additional files}

Additional file 1: Table S1. Sequences of primers used for vector construction.

Additional file 2: Table S2. Sequences of primers used for qRT-PCR.

Additional file 3: Figure S1. Partial gas chromatogram of C. psychrerythraea $34 \mathrm{H}$. A. FAMEs prepared from standards $C 16: 1^{\Delta 9 t}, C 16: 1^{\Delta 6 c}, C 16: 1^{\Delta 9 c}$ in an approximated ration of 7:4:10. B. Three major C16:1 peaks eluted at the corresponding retention time of $\mathrm{C} 16: 1^{\Delta 9 t}, \mathrm{C} 16: 1^{\Delta 6 c}, \mathrm{C} 16: 1^{19 c}$ standards, with shoulder peak appearing after $\mathrm{C} 16: 1^{\Delta 9 C}$. GC-MS analysis confirmed these peaks were $C 16: 1^{\Delta \theta t}, C 16: 1^{\Delta 7 c}, C 16: 1^{\Delta 9 c}, C 16: 1^{\Delta 11 c}$.

Additional file 4: Figure S2. Mass spectra of four C16:1 peaks in order, at retention time of 5.975, 6.050, 6.150 and 6.275 min.

Additional file 5: Figure S3. Partial gas chromatogram of C. psychrerythraea $34 \mathrm{H}$. A. FAMEs prepared from standards $\mathrm{C} 18: 1^{\Delta \mathrm{gt}}, \mathrm{C} 18: 1^{\Delta 6 \mathrm{C}}$ in an approximated ration of 1:10. B. Four C18:1 peaks eluted at the corresponding retention time of $C 18: 1^{\Delta 9 t}, C 18: 1^{\Delta 6 c}, C 18: 1^{\Delta 9 c}, C 18: 1^{\Delta 11 c}$ standards. GC-MS analysis confirmed these peaks were $\mathrm{C} 18: 1^{\Delta 9 t}, \mathrm{C} 18: 1^{\Delta t c}, \mathrm{C} 18: 1^{\Delta 9 c}$, C18:1 $111 \mathrm{C}$

Additional file 6: Figure S4. Mass spectra of 3-hydroxyl fatty acid methyl esters. A. 3OH-C10:0 FAME at $5.025 \mathrm{~min}(\mathrm{M}+202) ; \mathrm{B} .3 \mathrm{OH}-\mathrm{C} 12: 0$ FAME at $6.992 \mathrm{~min}(\mathrm{M}+230)$; C. 3OH-C14:0 FAME at $9.275 \mathrm{~min}(\mathrm{M}+258)$

Additional file 7: Figure S5. Mass spectra of TMS derivative hydroxy fatty acid methyl esters and their TMS derivatives. A. 3OH-C12:0 FAME $(M+302) ;$ B. 3OH-C14:0 FAME $(M+330)$.

Additional file 8: Figure S6. Mass spectra of branch chain fatty acid methyl esters. A. C14:0 FAME at 4.075 min (M + 242); B. 12-methyl-C14:0 FAME at $4.600 \mathrm{~min}(\mathrm{M}+256) ;$ C. C15:0 FAME at $4.833 \mathrm{~min}(\mathrm{M}+256)$. The different ratio of mass ion between $\mathrm{m} / \mathrm{z} 199$ and 213 in $B$ and $C$ suggested $B$ could be the anteiso isomer of C15:0 FAME, i.e., 13-methyl-C14:0.

Additional file 9: Figure S7. Mass spectra of polyunsaturated fatty acid methyl esters C20:4, C20:5, and C22:5.

Additional file 10: Figure S8. Statistical analysis of differential expressed genes after cerulenin treatment.

Additional file 11: Table S3. Analysis of C. psychrerythraea pfa gene expression by RNA-seq and qRT-PCR.

Additional file 12: Table S4. Differentially expressed genes in C. psychrerythraea treatment without or with $12 \mu \mathrm{g} / \mathrm{mL}$ cerulenin at $10^{\circ} \mathrm{C}$.

Additional file 13: Figure S9. Selective down-regulation of oxidation pathway genes. Bold indicated the gene had higher expression level in cerulenin-treated sample (M10_C) than in untreated sample (M10).

\section{Authors' contributions}

$X W, Y G, F H$ and GM conceived the study, XW, YP and GM conducted experiments. XW, YP, GM and XZ analyzed the data and wrote the manuscript. All authors read and approved the final manuscript.

\section{Author details}

1 Key Laboratory of Biology and Genetic Improvement of Oil Crops, Ministry of Agriculture, Oil Crops Research Institute of Chinese Academy of Agricultural Sciences, Wuhan 430062, China. ${ }^{2}$ Hubei Key Laboratory of Lipid Chemistry and Nutrition, Wuhan 430062, China. ${ }^{3}$ CSIRO Agriculture, Canberra, ACT 2601, Australia. ${ }^{4}$ CSIRO Food and Nutrition, Canberra, ACT 2601, Australia.
${ }^{5}$ Departamento de Biología Molecular e Instituto de Biomedicina y Biotecnología de Cantabria (IBBTEC), Universidad de Cantabria-CSIC, C/Albert Einstein 22, 39011 Santander, Spain.

\section{Acknowledgements}

We are grateful to Dr. Juan Maria Garcia-Lobo and Dr. Maria Cruz Rodriguez for RNA-Seq analysis performed in the massive sequencing service at the IBBTEC. This work was supported by the National Natural Science Foundation of China (3100078) and 948 project (2015-Z3) to X Wan and by Grants BIO2010-14809 and BFU2014-55534-C2-2-P from the Spanish Ministry of Economy and Competitiveness to GM.

\section{Competing interests}

The authors declare that they have no competing interests.

Received: 25 October 2015 Accepted: 25 January 2016

Published online: 06 February 2016

\section{References}

1. Swanson D, Block R, Mousa SA. Omega-3 fatty acids EPA and DHA: health benefits throughout life. Adv Nutr. 2012;3:1-7.

2. Friedrichs W, Ruparel SB, Marciniak RA, deGraffenried L. Omega-3 fatty acid inhibition of prostate cancer progression to hormone independence is associated with suppression of mTOR signaling and androgen receptor expression. Nutr Cancer. 2011;63:771-7.

3. Valenzuela R, Bascunan K, Chamorro R, Barrera C, Sandoval J, Puigrredon C, Parraguez G, Orellana P, Gonzalez V, Valenzuela A. Modification of docosahexaenoic acid composition of milk from nursing women who received alpha linolenic acid from chia oil during gestation and nursing. Nutrients. 2015;7:6405-24.

4. Xue Z, Sharpe PL, Hong SP, Yadav NS, Xie D, Short DR, Damude HG, Rupert RA, Seip JE, Wang J, et al. Production of omega-3 eicosapentaenoic acid by metabolic engineering of Yarrowia lipolytica. Nat Biotechnol. 2013;31:734-40

5. Gong Y, Wan X, Jiang M, Hu C, Hu H, Huang F. Metabolic engineering of microorganisms to produce omega-3 very long-chain polyunsaturated fatty acids. Prog Lipid Res. 2014;56:19-35.

6. Ruiz-Lopez N, Haslam RP, Usher S, Napier JA, Sayanova O. An alternative pathway for the effective production of the omega-3 long-chain polyunsaturates EPA and ETA in transgenic oilseeds. Plant Biotechnol J. 2015. doi:10.1111/pbi.12328.

7. Ruiz-Lopez N, Haslam RP, Napier JA, Sayanova O. Successful high-leve accumulation of fish oil omega-3 long-chain polyunsaturated fatty acids in a transgenic oilseed crop. Plant J. 2014;77:198-208.

8. Guo B, Jiang M, Wan X, Gong Y, Liang Z, Hu C. Identification and heterologous expression of a delta4-fatty acid desaturase gene from Isochrysis sphaerica. J Microbiol Biotechnol. 2013;23:1413-21.

9. Ujihara T, Nagano M, Wada H, Mitsuhashi S. Identification of a novel type of polyunsaturated fatty acid synthase involved in arachidonic acid biosynthesis. FEBS Lett. 2014;588:4032-6.

10. Shulse CN, Allen EE. Widespread occurrence of secondary lipid biosynthesis potential in microbial lineages. PLoS One. 2011;6:e20146. doi:10.1371/ journal.pone.0020146.

11. Shulse CN, Allen EE. Diversity and distribution of microbial long-chain fatty acid biosynthetic genes in the marine environment. Environ Microbiol. 2011;13:684-95.

12. Okuyama H, Orikasa Y, Nishida T, Watanabe K, Morita N. Bacterial genes responsible for the biosynthesis of eicosapentaenoic and docosahexaenoic acids and their heterologous expression. Appl Environ Microbiol. 2007;73:665-70

13. Napier JA. Plumbing the depths of PUFA biosynthesis: a novel polyketide synthase-like pathway from marine organisms. Trends Plant Sci. 2002;7:51-4.

14. Metz JG, Roessler P, Facciotti D, Levering C, Dittrich F, Lassner M, Valentine R, Lardizabal K, Domergue F, Yamada A, et al. Production of polyunsaturated fatty acids by polyketide synthases in both prokaryotes and eukaryotes. Science. 2001;293:290-3. 
15. Methe BA, Nelson KE, Deming JW, Momen B, Melamud E, Zhang X, Moult J, Madupu R, Nelson WC, Dodson RJ, et al. The psychrophilic lifestyle as revealed by the genome sequence of Colwellia psychrerythraea $34 \mathrm{H}$ through genomic and proteomic analyses. Proc Natl Acad Sci USA. 2005;102:10913-8.

16. Hashimoto M, Orikasa Y, Hayashi H, Watanabe K, Yoshida K, Okuyama H. Occurrence of trans monounsaturated and polyunsaturated fatty acids in Colwellia psychrerythraea strain 34H. J Basic Microbiol. 2015;55:838-45.

17. Trajtenberg F, Altabe S, Larrieux N, Ficarra F, de Mendoza D, Buschiazzo A, Schujman GE. Structural insights into bacterial resistance to cerulenin FEBS J. 2014;281:2324-38.

18. Price AC, Choi KH, Heath RJ, Li Z, White SW, Rock CO. Inhibition of betaketoacyl-acyl carrier protein synthases by thiolactomycin and cerulenin. Struct Mech J Biol Chem. 2001;276:6551-9.

19. Morita N, Nishida T, Tanaka M, Yano Y, Okuyama H. Enhancement of polyunsaturated fatty acid production by cerulenin treatment in polyunsaturated fatty acid-producing bacteria. Biotechnol Lett. 2005;27:389-93.

20. Allen EE, Facciotti D, Bartlett DH. Monounsaturated but not polyunsaturated fatty acids are required for growth of the deep-sea bacterium Photobacterium profundum SS9 at high pressure and low temperature. Appl Environ Microbiol. 1999;65:1710-20.

21. Zhou XR, Singh SP, Green AG. Characterisation of the FAD2 gene family from Hiptage benghalensis. A ricinoleic acid accumulating plant. Phytochemistry. 2013;92:42-8.

22. Fay $L$, Richli $U$. Location of double bonds in polyunsaturated fatty acids by gas chromatography-mass spectrometry after 4,4-dimethyloxazoline derivatization. J Chromatogr A. 1991;541:89-98.

23. Orikasa Y, Tanaka M, Sugihara S, Hori R, Nishida T, Ueno A, Morita N, Yano Y, Yamamoto K, Shibahara A, et al. pfaB products determine the molecular species produced in bacterial polyunsaturated fatty acid biosynthesis. FEMS Microbiol Lett. 2009;295:170-6.

24. Jiang H, Zirkle R, Metz JG, Braun L, Richter L, Van Lanen SG, Shen B. The role of tandem acyl carrier protein domains in polyunsaturated fatty acid biosynthesis. J Am Chem Soc. 2008;130:6336-7.

25. Kanehisa M, Goto S, Sato Y, Furumichi M, Tanabe M. KEGG for integration and interpretation of large-scale molecular data sets. Nucleic Acids Res. 2012;40:D109-14.

26. Kelley LA, Sternberg MJ. Protein structure prediction on the web: a case study using the Phyre server. Nat Protoc. 2009;4:363-71.

27. Romano D, Contente ML, Molinari F, Eberini I, Ruvutuso E, Sensi C, Amaretti A, Rossi M, Raimondi S. Recombinant S. cerevisiae expressing old yellow enzymes from non-conventional yeasts: an easy system for selective reduction of activated alkenes. Microb Cell Fact. 2014;13:60. doi:10.1186/1475-2859-13-60.
28. Quade N, Huo L, Rachid S, Heinz DW, Muller R. Unusual carbon fixation gives rise to diverse polyketide extender units. Nat Chem Biol. 2012;8:117-24.

29. White SW, Zheng J, Zhang YM, Rock. The structural biology of type II fatty acid biosynthesis. Annu Rev Biochem. 2005;74:791-831.

30. Fujita Y, Matsuoka H, Hirooka K. Regulation of fatty acid metabolism in bacteria. Mol Microbiol. 2007;66:829-39.

31. Allen EE, Bartlett DH. Structure and regulation of the omega-3 polyunsaturated fatty acid synthase genes from the deep-sea bacterium Photobacterium profundum strain SS9. Microbiology. 2002;148:1903-13.

32. Hauvermale A, Kuner J, Rosenzweig B, Guerra D, Diltz S, Metz JG. Fatty acid production in Schizochytrium sp.: involvement of a polyunsaturated fatty acid synthase and a type I fatty acid synthase. Lipids. 2006;41:739-47.

33. Schujman GE, Choi KH, Altabe S, Rock CO, de Mendoza D. Response of Bacillus subtilis to cerulenin and acquisition of resistance. J Bacteriol. 2001;183:3032-40.

34. Campbell JW, Cronan JE Jr. The enigmatic Escherichia coli fadE gene is yafH. J Bacteriol. 2002;184:3759-64.

35. Cuthbertson L, Nodwell JR. The TetR family of regulators. Microbiol Mol Biol Rev. 2013;77:440-75.

36. Agari Y, Agari K, Sakamoto K, Kuramitsu S, Shinkai A. TetR-family transcriptional repressor thermus thermophilus FadR controls fatty acid degradation. Microbiology. 2011;157:1589-601.

37. Zhang F, Ouellet M, Batth TS, Adams PD, Petzold CJ, Mukhopadhyay A, Keasling JD. Enhancing fatty acid production by the expression of the regulatory transcription factor FadR. Metab Eng. 2012;14:653-60.

38. Zhang $H$, Zheng B, Gao R, Feng Y. Binding of Shewanella FadR to the fabA fatty acid biosynthetic gene: implications for contraction of the fad regulon. Protein Cell. 2015;6:667-79.

39. Brige A, Van den Hemel D, Carpentier W, De Smet L, Van Beeumen JJ. Comparative characterization and expression analysis of the four old yellow enzyme homologues from Shewanella oneidensis indicate differences in physiological function. Biochem J. 2006;394:335-44.

40. Ryan J, Farr H, Visnovsky S, Vyssotski M, Visnovsky G. A rapid method for the isolation of eicosapentaenoic acid-producing marine bacteria. J Microbiol Methods. 2010;82:49-53.

41. Tilay A, Annapure U. Novel simplified and rapid method for screening and isolation of polyunsaturated fatty acids producing marine bacteria. Biotechnol Res Int. 2012;2012:542721.

\section{Submit your next manuscript to BioMed Central and we will help you at every step:}

- We accept pre-submission inquiries

- Our selector tool helps you to find the most relevant journal

- We provide round the clock customer support

- Convenient online submission

- Thorough peer review

- Inclusion in PubMed and all major indexing services

- Maximum visibility for your research

Submit your manuscript at www.biomedcentral.com/submit
() BioMed Central 